\title{
PSO and ANN Based Hybrid MPPT Algorithm for Photovoltaic Array under Partial Shading Condition
}

\author{
Md. Motakabbir Rahman, Md. Shahidul Islam*
}

Department of Electrical and Electronic Engineering, Rajshahi University of Engineering \& Technology (RUET), Rajshahi, BANGLADESH

${ }^{*}$ Corresponding Contact:

Email: siruet@gmail.com

\begin{abstract}
MPPT is an electronics device that extracts maximum available power from a PV module under varying environmental conditions. But most of the conventional MPPT methods fail to track maximum power under partial shading condition (PSC). Partial shading is the most common situation in PV power generation, which is caused if part of the series-connected strings is partially shaded. This situation leads to the multiple peaks in the P-V characteristics curve of the PV system. So stochastic search method, Particle Swarm Optimization (PSO), is used instead of the conventional methods to track maximum power under PSC. But the PSO method has the limitation of slow operation. So in this paper, a fast hybrid method is presented, which combines the PSO method with the ANN method. In this hybrid method, the ANN enables the existing PSO method to track MPP quickly by providing more accurate initial particle positions of the PSO algorithm.
\end{abstract}

Key words:

Photovoltaic (PV), Maximum power point tracking (MPPT), Partial Shading Condition (PSC), Particle Swarm Optimization (PSO), Artificial neural network (ANN)

\section{INTRODUCTION}

Renewable energy is becoming more and more popular as energy sources, as they are economically sustainable and environmentally reliable. People are keener to use renewable sources for power generation to reduce $\mathrm{CO}_{2}$ emission. Among these renewable sources, PV systems are becoming the first choice of people because they are cheap and easy to implement. Also, the development and improvement of the DC micro-grid and the Nanogrid system are providing the opportunity to spread electricity to the rural area. Now a day, not only the rural areas but also the cities are shifting toward a sustainable and environmentally friendly solar system (Abdullah et al., 2018). 
But the nonlinear behavior of the photovoltaic system under varying environmental conditions possesses challenges for designing the PV system. To overcome this problem, different MPPT methods are utilized, and they are making it possible to track maximum power under varying environmental conditions (Enslin, 1990). Some of those conventional methods are $\mathrm{P} \& \mathrm{O}$ and Incremental conductance methods. Some intelligent methods are also used for this tracking purpose, i.e., ANN and Fuzzy logic (Esram and Chapman, 2007; Elobaid et al., 2015). But there exists another environmental limitation of designing MPPT for the PV system, which is partial shading. This is one of the most common environmental disturbances which arises when some of the PV strings are partially shaded. As an effect of partial shading hotspot problem arises, which may damage the partially shaded strings Under PSC. So to eliminate the effect of partial shading bypass diode is used in parallel to each PV module. Due to the introduction of bypass diode across the PV cell multiple local maxima problem arises. Because of these multiple peaks, many of the conventional methods fail to track true MPP under partial shading (Islam et al., 2016).

Under PSC, when other conventional methods and intelligent method fails to track actual MPP, stochastic search method (Particle Swarm Optimization) provide quite an accurate searching record (Liu et al., 2012; Yunliang and Nan, 2015; Yang et al., 2017; Zheng et al., 2016). The main disadvantages of the PSO method are slow operation and large oscillations. To overcome large oscillation problems, several modifications were performed on the PSO method (Ishaque et al., 2012; Merchaoui, 2018; Yang et al., 2019; Mirhassani et al., 2014; Obukhov et al., 2020). Some modifications were also made on the reinitiating of the PSO algorithm. But the standalone PSO method was unable to detect the MPP fast. So faster and reliable method was needed to overcome this limitation. As the PSO was highly reliable under PSC but slow, modifications were made by including other methods in parallel with the PSO method in a hybrid structure.

Manickam et al. (2016) proposed a hybrid method that combined PSO with the P\&O method. In this method, the convergence time of the PSO reduced from 3.75 seconds to $500 \mathrm{~ms}$ as the search window is narrowed. But the further reduction of tracking time was desired. The artificial intelligent systems were necessary to be introduced as they were fast and have the capability of dealing with the nonlinearities in the I-V characteristics of PV systems. El-Helw et al. (2017) combined the P\&O method with ANN. As both ANN and $\mathrm{P} \& \mathrm{O}$ had a fast-tracking ability, this method was fast. But the method was unreliable under PSC because of multiple peaks. Bataineh and Eid (2018) combined a fuzzy logic controller with the P\&O method. Priyadarshi et al. (2020) proposed another hybrid method; they combined the ANFIS and PSO method under fluctuating sun irradiance. As the PSO method is reliable, the insertion of ANFIS made this method fast and reliable.

One of the most popular artificial intelligent systems is an Artificial Neural Network. The popularity of ANN is growing day by day because of its simplicity and easy implementation. To develop MPPT for the PV system under PSC, ANN can play a significant role. ANN predicts the non-linear behavior of the PV system more accurately. So the implementation of ANN with the PSO method in combination will improve the speed of tracking (Bataineh and Eid, 2018; Ngan and Tan, 2011). Also, due to the presence of the PSO algorithm, this method can detect MPP more reliably than other hybrid methods. In this paper, a hybrid method of ANN and PSO will be discussed. Also, the performance of the new hybrid method will be evaluated by comparing it with the standalone PSO method. Both the PSO and hybrid method will be applied to the same PV array configuration. Also, the applied irradiance pattern will be the same for both cases. 


\section{Partial Shading Condition}

The output voltage of a single PV module is low compared to the requirement for the system. To increase the voltage of the system, PV modules are connected in series. However, not all the PV modules of the array receive the same amount of irradiance all the time. When parts of the PV strings are partially shaded by heavy clouds or buildings or trees, the receiving amount of irradiance varies module to module.
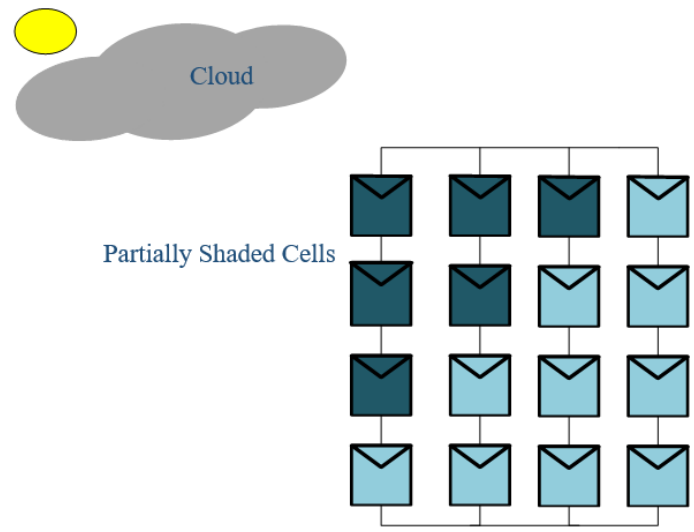

Fig. 1. Representation of partial shading situation



Fig. 2. PV array with bypass diode

This partial shading condition leads to significant power loss in the PV system. As there is an uneven distribution of sunlight among the strings, it creates a hot spot problem in the location of shaded cells. Only the fully illuminated PV cells generate a large amount of energy, while the shaded cells absorb this energy and convert it into heat. This situation is called the hot-spot problem, which may damage the partially shaded PV cells and consequently reduces the lifespan of the PV module. To eliminate the effect of partial shading, bypass diodes are inserted across each PV strings shown in fig.2.

Under partially shaded conditions, the short circuit current of each series-connected PV module may differ from each other. This leads the bypass diode of the partially shaded cell to be forward biased. And current flows through the bypass diode to prevent hot spots.

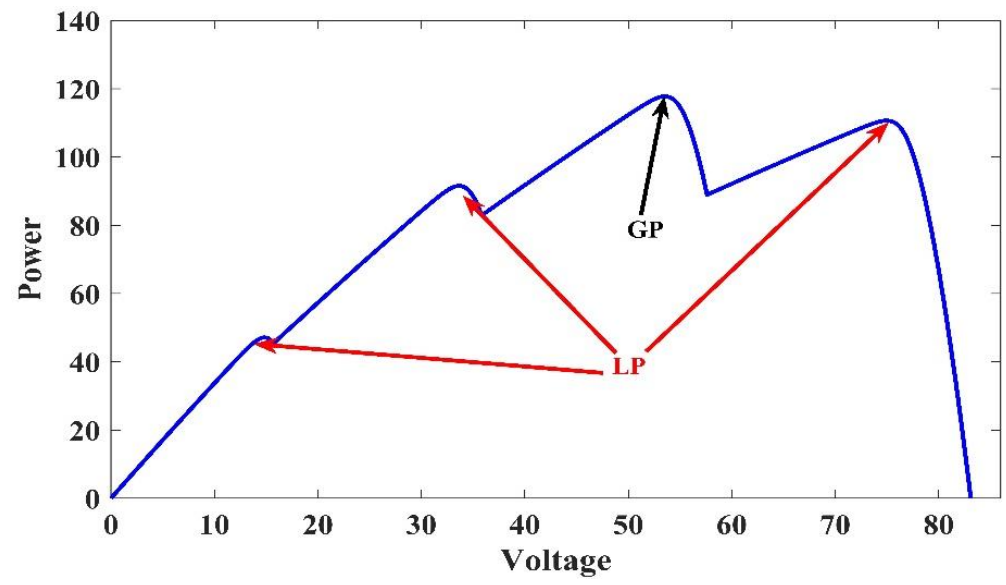

Fig. 3. P-V curve of PV array with bypass diode 
However, the insertion of the bypass diode causes another problem. It arises multiple peaks, namely global peak (GP) and local peak (LP), in which the only GP is the true MPP on P-V characteristic curve. The characteristics curve of a partially shaded PV array with a bypass diode is shown in fig. 3 .

As a result of multiple peaks, conventional methods, i.e., PSO, IncCond, and ANN, fail to track true global peak and lingers around the local peaks. So different MPP search approach is required, which can detect the global peak instead of lingering around the local peaks.

\section{MPPT Methods Under PaRTIAL SHAding}

Under partial shading condition, the conventional algorithm (P\&O, IncCond \& ANN) are not intelligent enough to differentiate among the global peak and local peak. So stochastic search method (PSO) is usually employed under PSC, which detects MPP accurately. A new PSO \& ANN based hybrid intelligent method can be applied under PSC, which will reduce the searching time. Both the PSO \& new hybrid will be discussed and compared here.

\section{Particle Swarm Optimization}

Due to several local peaks under partial shading, a stochastic search method named Particle swarm optimization (PSO) is employed to detect the global peak, which is the MPP of the array. The PSO is an optimization technique having a population or several particles. Initially, these particles are randomly distributed in a given search space based on some equations. And finally, these particles move toward the actual MPP and finds the actual solution. It is an effective technique that can be used to multi-variable function optimization with multiple local points, in this case, as an MPPT technique.

The PSO technique has two operators, one of them is velocity update (1), and another is particle position update (2). Both the velocity update and best particle position is dependent upon the power output of the PV module. And the output of this algorithm is the duty cycle for the switch of the boost converter. So the particle position defined the duty cycle and denoted by $\mathrm{D}$ in equation 2 . The two expressions are given below,

$$
\begin{aligned}
& v_{i}^{k+1}=w v_{i}^{k}+c_{1} r_{1}\left(p_{\text {besti }}-S_{i}^{k}\right)+c_{2} r_{2}\left(g_{\text {besti }}-S_{i}^{k}\right) \\
& D_{i}^{k+1}=D_{i}^{k}+v_{i}^{k+1}
\end{aligned}
$$

Where,

$v_{i}^{k}=$ current velocity vector

$v_{i}^{k+1}=$ modified velocity vector

$D_{i}^{k}=$ current position vector

$D_{i}^{k+1}=$ modified position vector

$w=$ inertia weight

$p_{\text {best }}=$ best position found by particle $i$

$g_{\text {best }}=$ best position found by particle group

$c_{1}=$ cognitive coefficient

$c_{2}=$ social coefficient

$r_{1}=$ random parameter

$r_{2}=$ random parameter

The PSO uses the M number of particles or agents to search for the maximum power for the PV array. The more particle improves the accuracy of search but increases the computational 
time or the tracking time. In this hybrid method, $M=3$ particles are chosen. Each of the particles moves in a given search space with a velocity, $\mathrm{v}_{\mathrm{i}}^{\mathrm{k}}$. Then, a new velocity value for each particle is calculated based on the current velocity, the previous best position, and the global best position, for each iteration. After that, a new position is updated by using the previous position of the particle and the new velocity value according to the previous two equations.

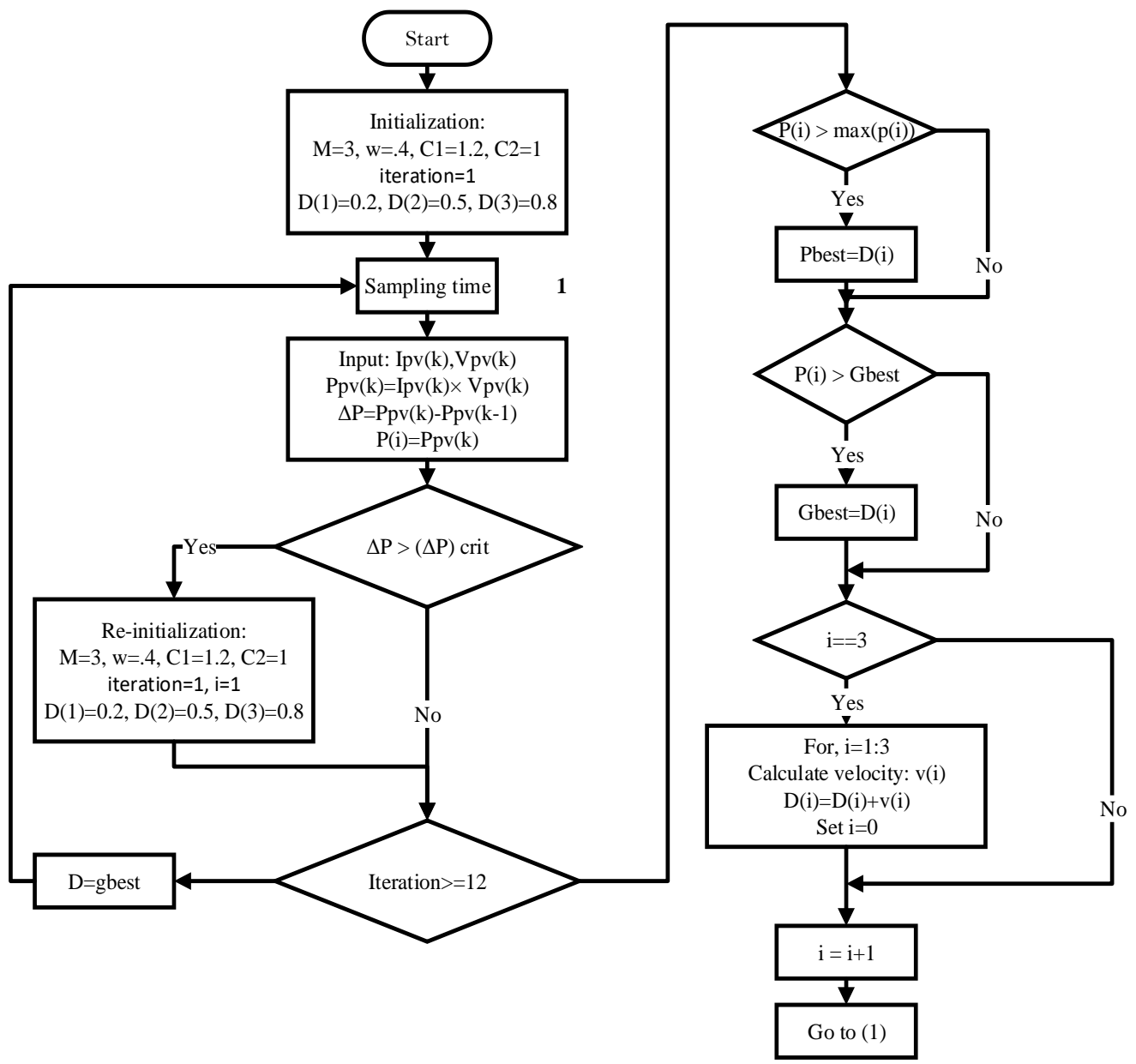

Fig. 4. Flow Chart of PSO Method

In this research work, three particles $(\mathrm{M}=3)$ were used. The initial velocities of all particles were set to zero. As the range of duty cycle is 0 to 1 , the initial positions of the particles denoted by D (Duty cycle) were selected as, $0.2,0.5 \& 0.8$. Three parameters of the PSO algorithm were needed to be calculated first, those are, the weight inertia (w), cognitive coefficient (c1), and the social coefficient (c2). These parameters were calculated by the trial and error process and they were selected as $0.4,1.2$, and 1, respectively.

As the irradiance pattern changes dynamically, the PSO algorithm must be reinitiated whenever there is an environmental change. This change can be detected by comparing the change of power with a critical value. When $\Delta \mathrm{P}>(\Delta \mathrm{P})_{\text {crit, }}$ the algorithm reinitiates itself to its initial value that was set. Here, $\Delta \mathrm{P}=\mathrm{P}^{\mathrm{k}}-\mathrm{P}^{\mathrm{k}-1}$. 


\section{PSO \& ANN-based Hybrid MPPT Method}

PSO is a stochastic search method, which takes a considerable amount of time to track a global peak. So some modifications were required. In the new ANN and PSO based hybrid method, the initial Particle position of particle Swarm Optimization (PSO) method is provided by an Artificial Neural Network (ANN). This initial particle position(Ic) is near the global MPP. So the range of the PSO algorithm is reduced. Using this initial value, the PSO algorithm detects the output current of the PV array at global MPP. And the PSO algorithm is now able to find the global MPP quickly.

Also, whenever there is a sudden change of solar irradiance, ANN detects the change \& provides a new initial particle position (Ic) for the PSO algorithm. The flow chart of this method is shown in fig. 4 .

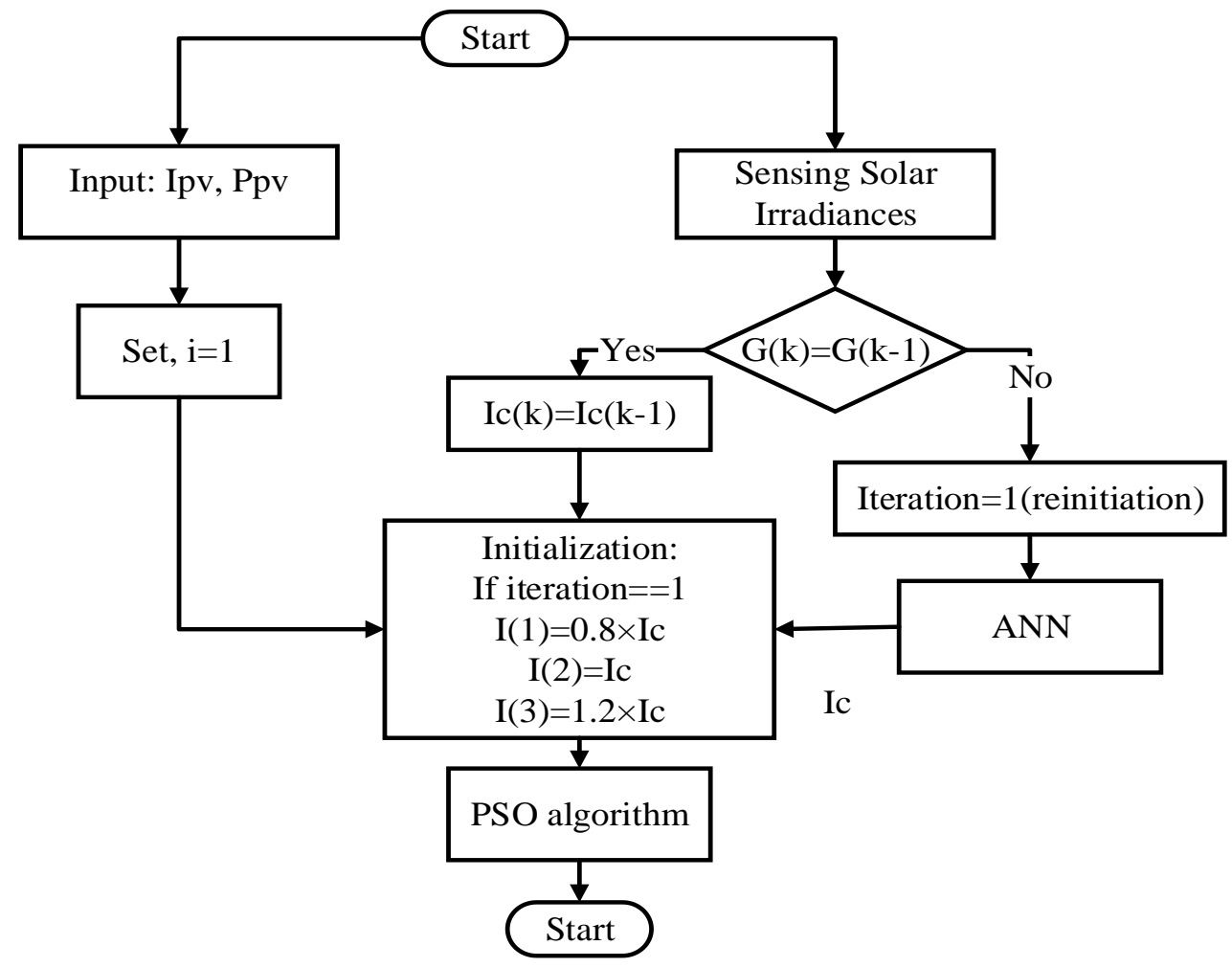

Fig. 5. Flow Chart of the Hybrid Method

In the PSO algorithm, there are three particles used, and one of their position (Ic) is provided by the ANN. So two more positions are selected, which are respectively $80 \% \& 120 \%$ of Ic. Thus these three-particle positions are providing the PSO algorithm a very narrow search space. The block diagram of the Hybrid method is shown in fig. 6. The ANN takes the irradiance pattern as input and provides initial particle positions to the PSO algorithm. Where the PSO algorithm tries to achieve MPP by varying the reference current toward IMPP. And a PID controller changes the duty cycle to control the boost converter to follow the reference current. 




Fig. 6. Block diagram of Hybrid Method

\section{MetHodology}

\section{PV Array Design:}

For describing the Partial shading situation, an array of PV panels is required. To carry out this research work, a SIMULINK model of the array is designed first. The array consists of four PV panels, which are connected in a series configuration. All of the PV cells have the same characteristics. Different irradiation patterns can be applied to this PV array, which can be used to emulate the practical PV array. Now using this array, we can test both PSO and proposed hybrid method. The parameters of a PV panel, used for SIMULINK modeling is given below.

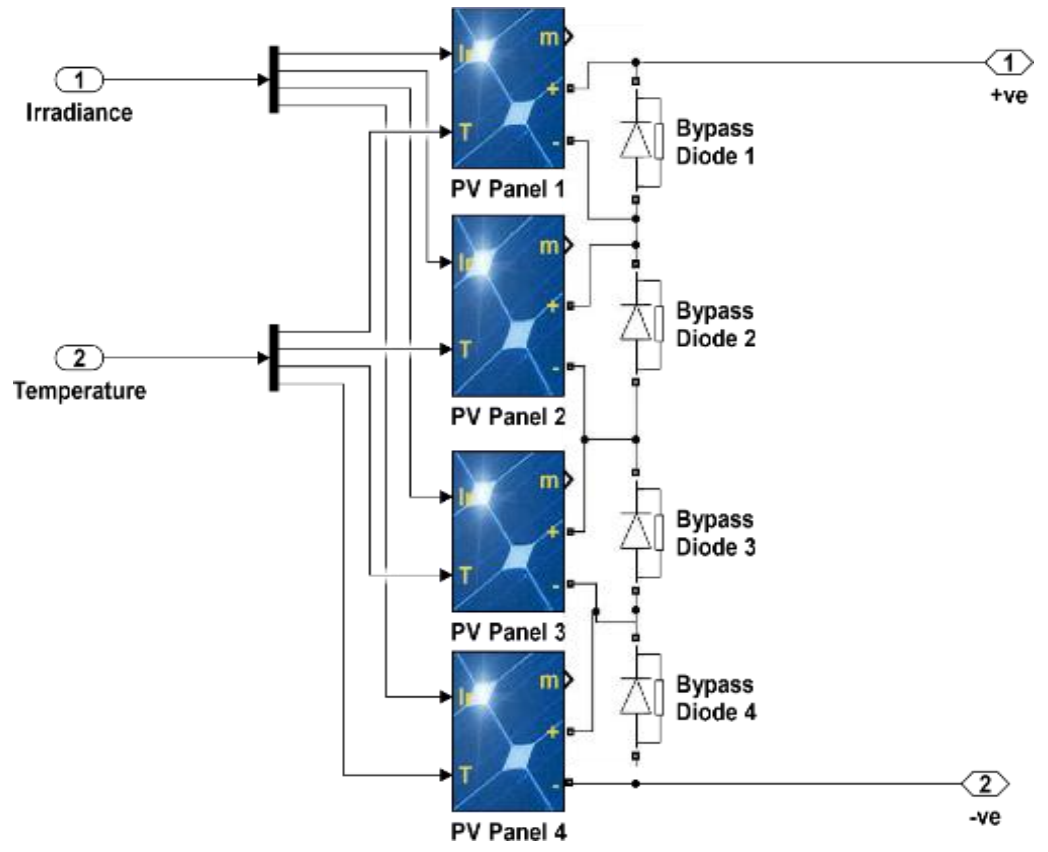

Fig. 7. PV array design 
Table 1. PV module parameters

\begin{tabular}{ll}
\hline Open circuit voltage $\mathrm{V}_{\mathrm{OC}}$ & $21.1 \mathrm{~V}$ \\
Short-circuit current Isc & $3.8 \mathrm{~A}$ \\
Cells per module $\left(\mathrm{N}_{\text {cell }}\right)$ & 36 \\
Temperature coefficient of $\mathrm{V}_{\mathrm{OC}}$ & $-0.229 \% /{ }^{\circ} \mathrm{C}$ \\
Temperature coefficient of Isc & $0.0307 \% /{ }^{\circ} \mathrm{C}$ \\
Maximum Power & $60.53 \mathrm{~W}$ \\
\hline
\end{tabular}

These panels are to be connected in a series configuration. But to protect the array from the hot spot problem, bypass diode is to be connected in parallel to each panel. The SIMULINK model of the PV array is shown below.

\section{Partial Shading Pattern}

For illustrating the partial shading situation, the array is to be illuminated by irradiance of different patterns. Here in this research work, two patterns are used (Pattern 1 \& Pattern 2), which are shown in the table below.

Table 2. Partial Shading Pattern

\begin{tabular}{ccc}
\hline Module No. & 0-1sec $\left(\mathrm{wm}^{-2}\right)$ & $1-2 \mathrm{sec}\left(\mathrm{wm}^{-2}\right)$ \\
\hline PV1 & 400 & 900 \\
PV2 & 700 & 800 \\
PV3 & 900 & 800 \\
PV4 & 900 & 900 \\
\hline
\end{tabular}

The P-V characteristic of the PV array for two different patterns is shown in the figure below.

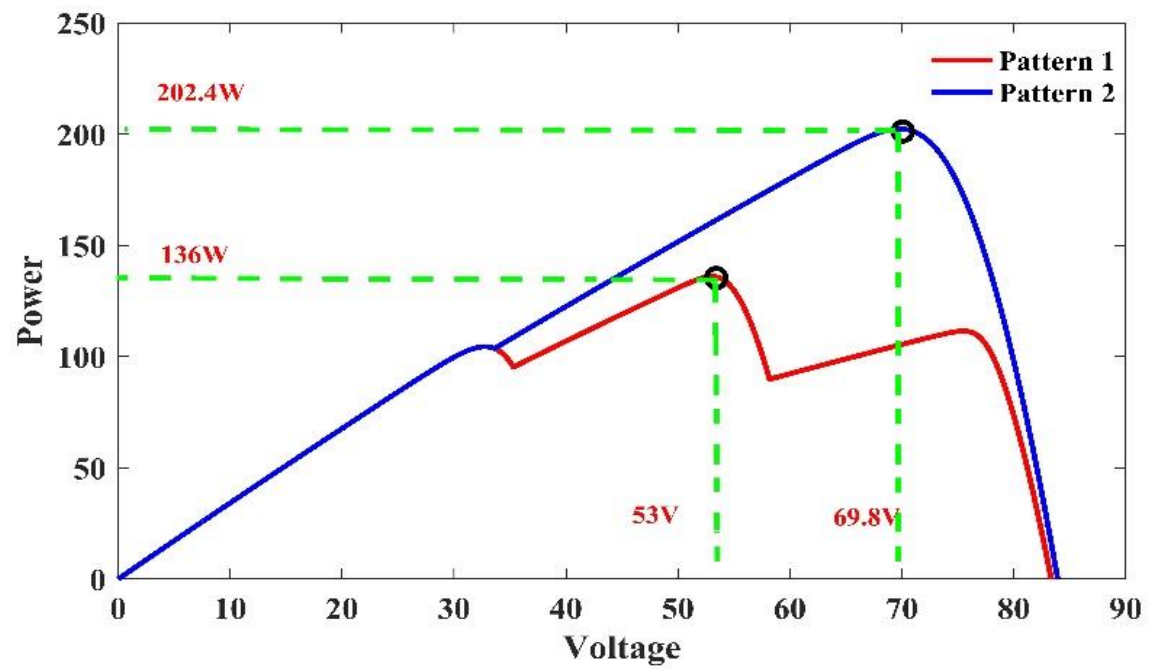

Fig. 8. Validation performance of ANN for hybrid 2 model

From the figure, the MPP for pattern- 1 is 136 Watt at $53 \mathrm{~V}$, and for pattern-2, the global peak is 202.4 Watt at $69.8 \mathrm{~V}$. There are several local peaks also at both patterns. But the MPPT algorithm should be able to detect the MPP while the irradiance pattern changes from pattern 1 to pattern 2, neglecting the local peaks. 


\section{ANN Training}

ANN mentioned in the hybrid model, takes irradiances in each PV panel of the array as input and generates Ic. As irradiance can be different on each of those modules, there are thousands of combinations of partial shading patterns can be formed in the array. Among those combinations, we took 100 sets of data from 100 shading patterns and used them for training the ANN. The ANN training parameters for this method are tabulated in table 3 . The ANN takes irradiance of 4 PV cells as input and generates Ic as output.

Table 3. ANN training Parameters

\begin{tabular}{lc}
\hline \multicolumn{1}{c}{ Parameters } & Values \\
\hline Network type & Feed forward back propagation \\
Number of layers & 2 layers \\
Number of neuron in the hidden layer & 15 neuron \\
Number of neurons output layer & 1 neuron \\
The Activation function of the hidden layer & tansig \\
The Activation function of the output layer & purelin \\
Network training function & trainlm \\
Show & 50 \\
Leaning rate & 0.05 \\
Epochs & 1000 \\
Training goal/ Error tolerance & $1 \mathrm{e}-3$ \\
\hline
\end{tabular}

The training performance of the ANN is shown in fig. 8 , and the validation performance is shown in fig. 9. From the validation curve, we can see at the best validation performance is 0.048906 at epoch 4 .
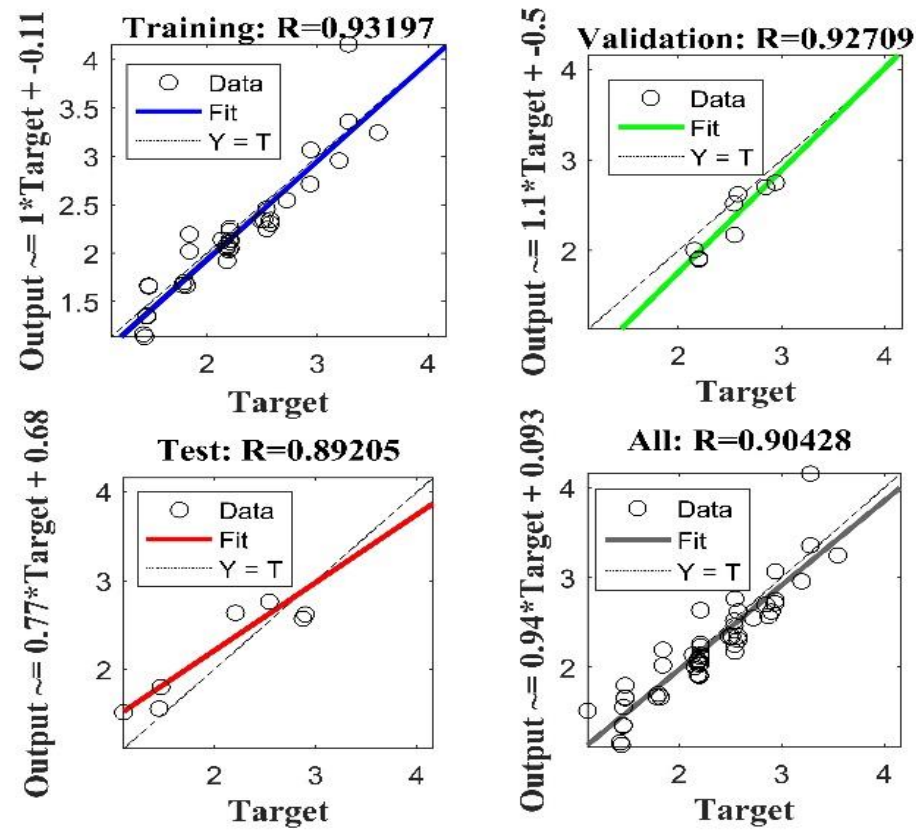

Fig. 8. Training performance of ANN for the hybrid model 




Fig. 9. Validation performance of ANN for the hybrid model

\section{Simulation Model}



Fig. 10. Simulink model of the Hybrid method

\section{Model Parameters}

In the simulation model of the proposed hybrid MPPT method, the array is connected across a boost converter. The converter extracts maximum power from the array under all conditions. The parameters of the boost converter tabulated below.

Table 4. Boost converter parameters

\begin{tabular}{ll}
\hline Parameters & Values \\
\hline C1 & $40 \times 10^{-6} \mathrm{~F}$ \\
L1 & $1 \times 10^{-3} \mathrm{~F}$ \\
C2 & $40 \times 10^{-6} \mathrm{~F}$ \\
R & $120 \Omega$ \\
\hline
\end{tabular}


The switch performs the main operation. The duty cycle of the switch is defined by the MPPT technique \& by the PID controller. And according to this duty cycle, the pulse generator generates a pulse for the switch. The switching frequency is $10 \mathrm{KHz}$. Also, in the proposed hybrid method, the PSO method is integrated with the ANN. The parameters for the PSO technique are shown below.

Table 5. PSO parameters

\begin{tabular}{ll}
\hline Parameters & Values \\
\hline Number of particles & 3 \\
Maximum Number of iterations & 12 \\
Weight $(w)$ & 0.4 \\
C1 & 1.2 \\
C2 & 2 \\
\hline
\end{tabular}

\section{RESULT}
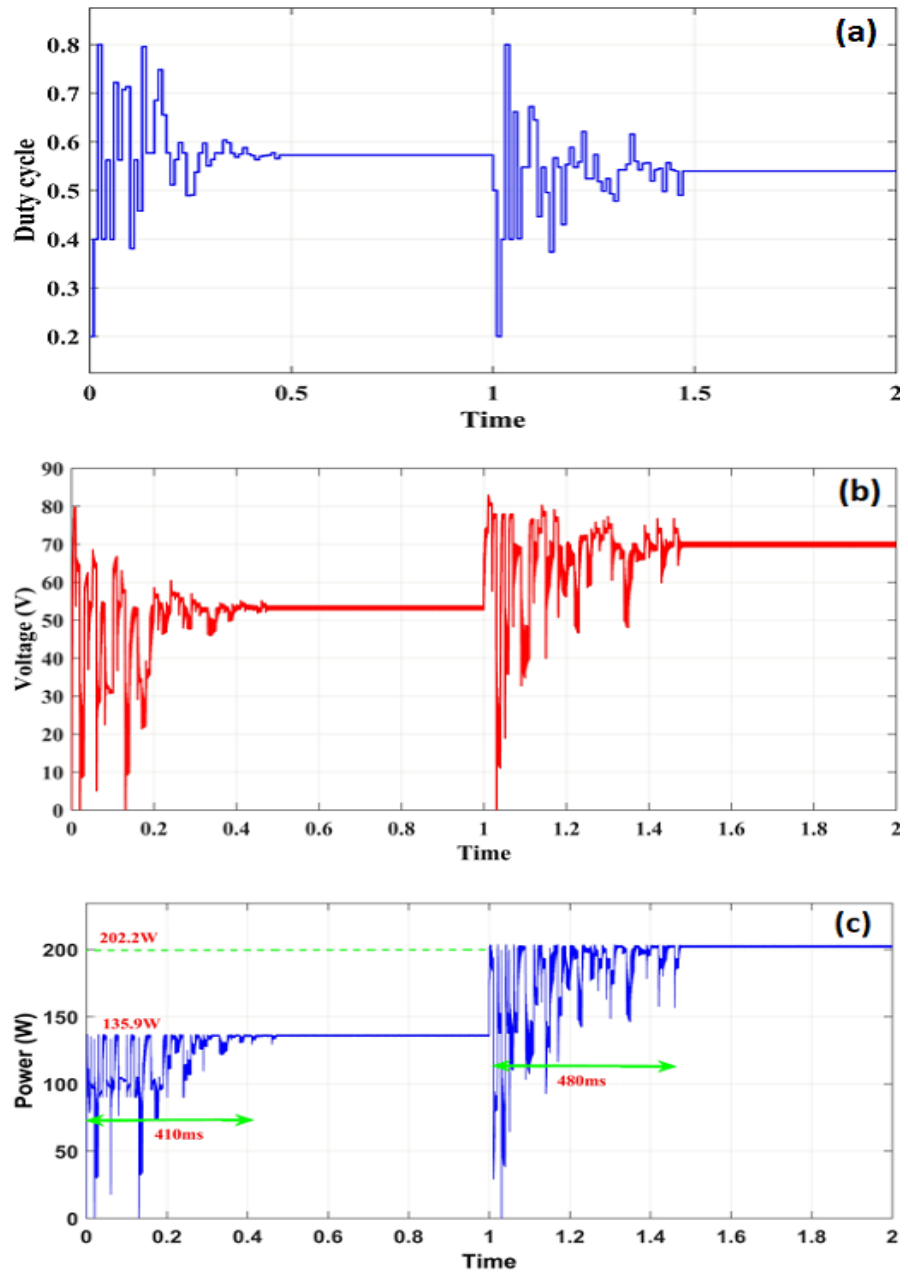

Fig. 11. (a) Duty cycle (b) PV current and (c) Output power of PV array using PSO method 
Firstly, the standalone PSO algorithm is used to test the speed \& efficiency of the method under the given partial shading pattern. This method can track maximum power with a great deal of efficiency. The tracking performance of a standalone PSO MPPT is shown below.

The tracking performance of the stand-alone PSO method is recorded in the table below, where the tracked power, efficiency, and the tracking time is shown.

Table 6. Tracking efficiency \& tracking time of the PSO method

\begin{tabular}{lll}
\hline Partial Pattern & Pattern 1 & Pattern 2 \\
\hline Maximum Power & $136 \mathrm{~W}$ & $202.4 \mathrm{~W}$ \\
Tracked Power & $135.9 \mathrm{~W}$ & $202.2 \mathrm{~W}$ \\
Efficiency & $99.92 \%$ & $99.90 \%$ \\
Tracking Time & $410 \mathrm{~ms}$ & $480 \mathrm{~ms}$ \\
\hline
\end{tabular}
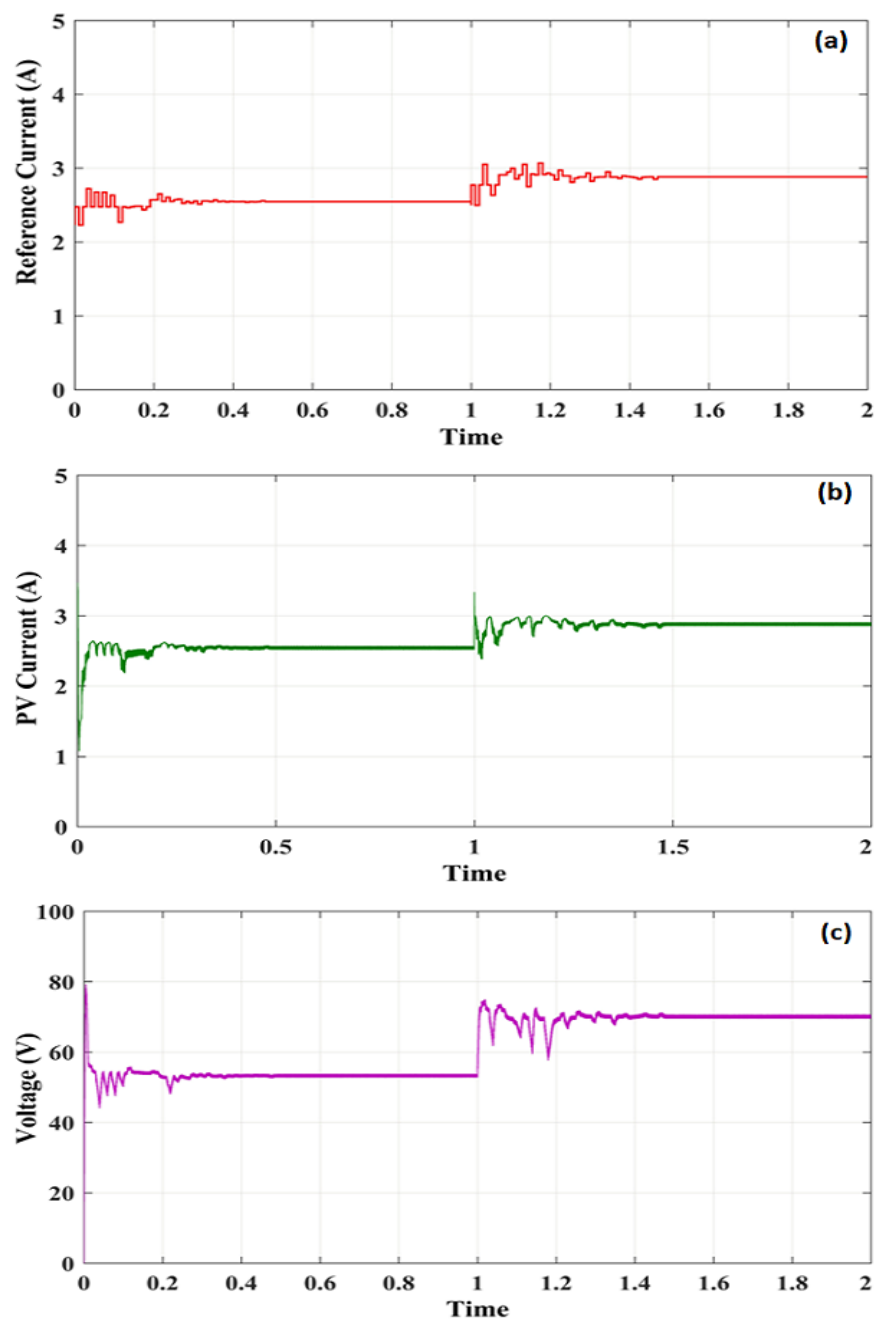

Fig. 12. (a) Reference current (PSO output) (b) PV current and (c) Output power of PV array under new hybrid method 
Analyzing the power curve, it is clear that this method can track MPP under PSC efficiently. Under dynamic environmental change from irradiance pattern 1 to pattern 2, the method reinitiated itself and searched the true MPP accurately within $480 \mathrm{~ms}$. But this method operates slowly due to large searching space. PSO takes time to search the true MPP in its search space. So there is a significant delay in this process, which is over $400 \mathrm{~ms}$.

Now MPP of the similar PV array is under the same irradiance pattern is tracked by the hybrid method to ensure whether the new method works faster or not. Firstly, the reference current is produced by the PSO algorithm is shown fig. 12(a). Secondly, the PID controlled works quickly and efficiently to follow the suggested reference current by the PSO algorithm. And the PV current is shown in fig. 12(b). Finally, as a result of ANN, PSO \& PID controller, the converter is capable of operating the PV array on optimal voltage, which are $53 \mathrm{~V}$ in the case of pattern 1 and $69 \mathrm{~V}$ for pattern 2 . These current and voltage wave shapes suggest that the new hybrid method can track MPP quickly and also with less ripple.

Now, the power curve of the PV array for this new hybrid method is shown in fig. 13. The power curve suggests that the new method can detect the maximum power of $135.9 \mathrm{~W}$ during pattern 1 within $220 \mathrm{~ms}$ and $202.1 \mathrm{~W}$ during pattern 2 within $210 \mathrm{~ms}$.

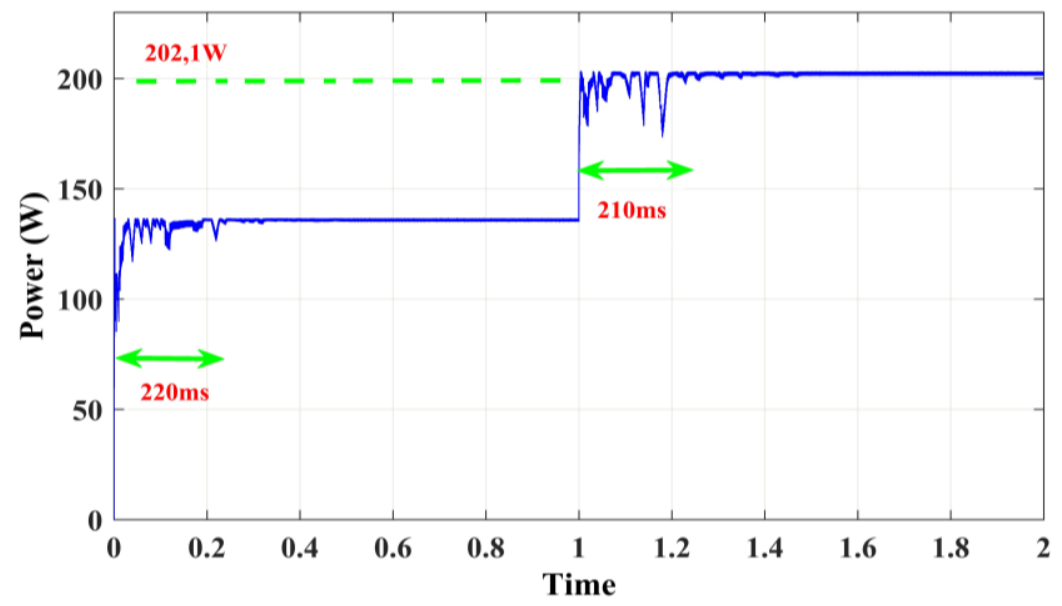

Fig. 13. Tracked PV power by the hybrid method

The performance of the new hybrid method under the changing environment is tabulated at the following table.

Table 7. Tracking efficiency \& tracking time of the hybrid method

\begin{tabular}{lcc}
\hline \multicolumn{1}{c}{ Shading Pattern } & Pattern 1 & Pattern 2 \\
\hline Maximum Power & $136 \mathrm{~W}$ & $202.4 \mathrm{~W}$ \\
Tracked Power & $135.9 \mathrm{~W}$ & $202.1 \mathrm{~W}$ \\
Efficiency & $99.92 \%$ & $99.89 \%$ \\
Tracking Time & $220 \mathrm{~ms}$ & $210 \mathrm{~ms}$ \\
\hline
\end{tabular}

While comparing performance between PSO and new hybrid method, we can see that there is a significant improvement in speed while tracking the MPP. The hybrid method is faster and efficient. 


\section{Conclusions}

This research paper introduced a new hybrid method, which combines both ANN \& PSO method which works efficiently under partial shading condition. Also, the performance of this hybrid method is compared with the standalone PSO method. The tracking time reduced significantly in the case of the hybrid method compared to the PSO method. But there is some scope of improvement for this method. Firstly, its speed can be further improved if the ANN is trained with more accurate data, which will reduce the mean square error. And thus, it will allow us to reduce the search space further for the PSO algorithm. Also, a more accurate ANN result can eliminate the need for a stochastic search (PSO) method, instead, we can use faster search algorithms like $\mathrm{P} \& \mathrm{O}$ and IncCond methods. Secondly, the ANN takes irradiance on the different modules as input, but the inaccuracy of those sensors can be the major problem for practical cases. The need for irradiance measuring sensors can be eliminated by detecting the irradiance pattern only by observing the PV current and voltage curve. To do that, more and more trained ANN is required. As there are thousands of possible shading pattern may occur, the ANN has to more accurate. Despite this, the tracking speed and efficiency of the proposed hybrid method lead to the belief that this method may provide some contributions to the development of solar energy.

\section{REFERENCES}

Abdullah, M. A., Al-Hadhrami, T., Tan, C. W., and Yatim, A. H. (2018). Towards Green Energy for Smart Cities: Particle Swarm Optimization Based MPPT Approach. In IEEE Access, 6, 58427-58438.

Bataineh, K., and Eid, N. (2018). A Hybrid Maximum Power Point Tracking Method for Photovoltaic Systems for Dynamic Weather Conditions. In MDPI AG, 2079-9276.

El-Helw, H. M., Magdy, A., and Marei, M. I. (2017). A Hybrid Maximum Power Point Tracking Technique for Partially Shaded Photovoltaic Arrays. In IEEE Access, 5, 11900-11908.

Elobaid, L. M., Abdelsalam, A. K., and Zakzouk, E. E. (2015). Artificial neural network-based photovoltaic maximum power point tracking techniques: a survey. In IET Renewable Power Generation, 9(8), 1043-1063.

Enslin, J. H. R. (1990). Maximum power point tracking: a cost saving necessity in solar energy systems. IECON '90: 16th Annual Conference of IEEE Industrial Electronics Society, Pacific Grove, CA, USA, 1073-1077.

Esram, T., and Chapman, P. L. (2007). Comparison of Photovoltaic Array Maximum Power Point Tracking Techniques. In IEEE Transactions on Energy Conversion, 22(2), 439-449.

Ishaque, K., Salam, Z., Amjad, M., and Mekhilef, S. (2012). An Improved Particle Swarm Optimization (PSO)-Based MPPT for PV with Reduced Steady-State Oscillation. In IEEE Transactions on Power Electronics, 27(8), 3627-3638.

Islam, M. R., Rahman, F, and Xu, W. (2016), Advances in Solar Photovoltaic Power Plants, Springer-Verlag Berlin Heidelberg. Accessed: Jun. 28, 2020. [Online]. Available: https://www.springer.com/series/8059

Liu, Y., Huang, S., Huang, J., and Liang, W. (2012). A Particle Swarm Optimization-Based Maximum Power Point Tracking Algorithm for PV Systems Operating Under Partially Shaded Conditions. In IEEE Transactions on Energy Conversion, 27(4), 1027-1035.

Manickam, C., Raman, G. R., Raman, G. P., Ganesan, S. I., and Nagamani, C. (2016). A Hybrid Algorithm for Tracking of GMPP Based on P\&O and PSO with Reduced Power Oscillation in String Inverters. In IEEE Transactions on Industrial Electronics, 63(10), 6097-6106.

Merchaoui, M., Sakly, A., and Mimouni, M. F. (2018). Improved fast particle swarm optimization based PV MPPT. 2018 9th International Renewable Energy Congress (IREC), Hammamet, 1-7. 
Mirhassani, S. M, Razzazan, M., and Ramezani, A. (2014). An improved PSO based MPPT approach to cope with partially shaded condition. 2014 22nd Iranian Conference on Electrical Engineering (ICEE), Tehran, 550-555.

Ngan, M. S., and Tan, C. W. (2011). A study of maximum power point tracking algorithms for standalone Photovoltaic Systems. 2011 IEEE Applied Power Electronics Colloquium (IAPEC), Johor Bahru, pp. 22-27.

Obukhov, S., Ibrahim, A., Zaki-Diab, A. A., Al-Sumaiti, A. S., and Aboelsaud, R. (2020). Optimal Performance of Dynamic Particle Swarm Optimization Based Maximum Power Trackers for Stand-Alone PV System Under Partial Shading Conditions. In IEEE Access, 8, 20770-20785.

Priyadarshi, N., Padmanaban, S., Holm-Nielsen, J. B., Blaabjerg, F., and Bhaskar, M. S. (2020). An Experimental Estimation of Hybrid ANFIS-PSO-Based MPPT for PV Grid Integration under Fluctuating Sun Irradiance. In IEEE Systems Journal, 14(1), 1218-1229.

Yang, H. Li, D., Su, W., Lü, J., and Yu, X. (2019). An Overall Distribution Particle Swarm Optimization MPPT Algorithm for Photovoltaic System under Partial Shading. In IEEE Transactions on Industrial Electronics, 66(1), 265-275.

Yang, Z., Duan, Q., Zhong, J., Mao, M., and Xun, Z. (2017). Analysis of improved PSO and perturb \& observe global MPPT algorithm for PV array under partial shading condition. 2017 29th Chinese Control and Decision Conference (CCDC), Chongqing, 549-553.

Yunliang, W., and Nan, B. (2015). Research of MPPT control method based on PSO algorithm. 2015 4th International Conference on Computer Science and Network Technology (ICCSNT), Harbin, 698-701.

Zheng, Y., Wang, W., Chen, W., and Li, Q. (2016). Research on MPPT of photovoltaic system based on PSO under partial shading condition. 2016 35th Chinese Control Conference (CCC), Chengdu, 86548659. 


\section{How to Cite:}

Rahman, M. M., \& Islam, M. S. (2020). PSO and ANN Based Hybrid MPPT Algorithm for Photovoltaic Array under Partial Shading Condition. Engineering International, 8(1), 9-24. https://doi.org/10.18034/ei.v8i1.481 